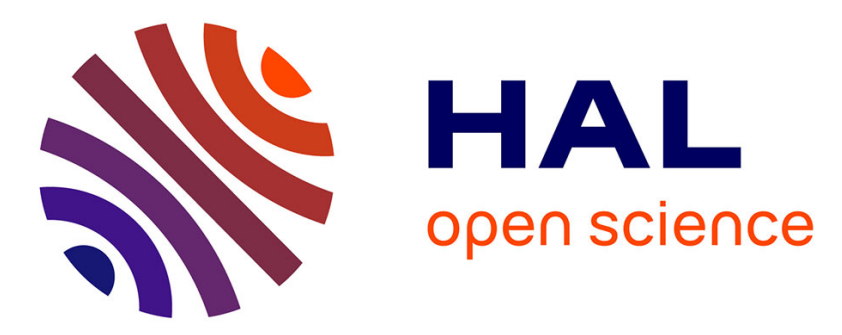

\title{
A Hybrid Control Strategy for Quadratic Boost Converters with Inductor Currents Estimation
}

\author{
Antonino Sferlazza, Carolina Albea-Sanchez, Germain Garcia
}

\section{To cite this version:}

Antonino Sferlazza, Carolina Albea-Sanchez, Germain Garcia. A Hybrid Control Strategy for Quadratic Boost Converters with Inductor Currents Estimation. Control Engineering Practice, 2020, 103, pp.104602. 10.1016/j.conengprac.2020.104602 . hal-02917476

\section{HAL Id: hal-02917476 https://hal.laas.fr/hal-02917476}

Submitted on 19 Aug 2020

HAL is a multi-disciplinary open access archive for the deposit and dissemination of scientific research documents, whether they are published or not. The documents may come from teaching and research institutions in France or abroad, or from public or private research centers.
L'archive ouverte pluridisciplinaire HAL, est destinée au dépôt et à la diffusion de documents scientifiques de niveau recherche, publiés ou non, émanant des établissements d'enseignement et de recherche français ou étrangers, des laboratoires publics ou privés. 


\title{
A Hybrid Control Strategy for Quadratic Boost Converters with Inductor Currents Estimation
}

\author{
Antonino Sferlazza ${ }^{\mathrm{a}}$, Carolina Albea-Sanchez ${ }^{\mathrm{b}}$, Germain Garcia ${ }^{\mathrm{b}}$ \\ ${ }^{a}$ University of Palermo, Department of Engineering, viale delle scienze Ed. 10, 90128 Palermo, Italy. E-mail: antonino. sferlazza@unipa.it \\ ${ }^{b}$ LAAS-CNRS, Université de Toulouse, CNRS, UPS, INSA, 7 avenue du Colonel Roche, 31031, Toulouse, France. E-mail: calbea@laas.fr, \\ germain.garcia@laas.fr
}

\begin{abstract}
This paper deals with a control strategy for a DC-DC quadratic boost converter. In particular, a hybrid control scheme is proposed to encompass a control law and an observer for the estimation of the system states, based only on the measurements of the input and output voltages. Differently from classical control methods, where the controller is designed from a small-signal model, here the real model of the system is examined without considering the average values of the discrete variables. Using hybrid dynamical system theory, asymptotic stability of a neighborhood of the equilibrium point is established, ensuring practical stability of the origin, which contains estimation and tracking errors. Experimental results show the effectiveness of the proposed approach.
\end{abstract}

Keywords: Quadratic boost converter, hybrid dynamical systems, switching systems, observer, linear matrix inequalities.

\section{Introduction}

Nowadays, the development of renewable power sources drives the interest towards DC-DC conversions, in particular to control DC-DC converters. Indeed, the most part of these sources are characterized by a low voltage output, i.e. photovoltaic panels, fuel cells, etc, as well as, the most part of storage systems, i.e. batteries supercapacitors, etc. This means that a DC-DC conversion stage is needed in order to allow the distribution of such energy (Carrasco et al., 2006). For this reason, a relevant effort has been done in order to increase both the efficiency of DC-DC converters and their steady-state gain. The improvement of the efficiency of these kind of systems is important, because it allows to reduce losses, such as switching and Joule losses. The steady-state gain is also relevant to reduce the number of conversion stages when a high conversion ratio is required. Together with these characteristics, that are strictly related to circuit topology and selected devices, it is very important to reduce:

- switching frequency,

- transient times, as well as, current and voltage overshoots,

- current ripples,

which are influenced by the selected control technique.

A converter particularly suitable for the applications mentioned above is the quadratic boost converter, because it presents a high voltage ratio. Among the existing configurations of these converters, it is possible to distinguish two main topologies. The first one consists in two independent boost stages with two switching devices (Chen et al., 2001) (in the following referred as $\mathrm{CBC}$ ), while the second one is composed of only one conversion stage with only one switching device (Baek et al., 2005; Zhao and Lee, 2003) (in the following referred as QBC). Multi-stage configurations can be also found, which achieve a very high input-output ratio (Luo and Ye, 2004). There are some works in the literature, where QBC and CBC are compared. For example, Choudhury and Nayak (2015) shows that both of them can deliver a high voltage gain, but the stress in QBCs is higher, increasing the cost of these converters. Nevertheless, the switching losses for the QBC are reduced with respect to the $\mathrm{CBC}$, allowing to obtain a higher efficiency.

In García-Vite et al. (2017), the authors propose a QBC that allows to increase the voltage gain and to drain the ripple-free current from the source. These features have a special interest in renewable energy systems, that are composed of low voltage generation sources and are sensitive to current ripples, such as in fuel cells applications. Other works related with the design of QBC with low current and low voltage stress for fuel-cell applications are presented in Al-Saffar and Ismail (2015); Wang (2018). Moreover, in Morales-Saldaña et al. (2014), the authors provide a QBC based on the reduced redundant power processing $\left(R^{2} P^{2}\right)$ principle, as well as, on a controller design methodology using current-programmed control satisfying the specifications about output voltage regulation. Chen et al. (2016) shows a QBC with a higher Power Factor Correction (PFC), obtained from a variable duty-cycle control. Alonge et al. (2017) proposes a transformer-less single switch topology of QBC. In Patidar and Umarikar (2015), the authors deal with QBCs for micro-inverter applications that integrate a high efficiency in the step-up capabilities.

Focusing on the control techniques adopted for the QBC, it is useful to mention some non-standard robust control solutions (Olalla et al., 2011). For example, a double loop sliding-mode controller is proposed in Lopez-Santos et al. (2015). In partic- 
ular, the proposed controller presents an inner loop based on sliding-mode control, whose sliding surface is defined for the input inductor current. The current reference value of the sliding surface is modified by a Proportional-Integral (PI) compensator in an outer loop that operates over the output voltage error. López-Santos et al. (2013) presents an efficiency analysis for such converters, while an interesting description of the steadystate behavior is shown in Lopez-Santos et al. (2017). Note that the idea of a double control loop, with an inner current loop, is often applied by using linear averaged models to design a controller under peak current-mode control (Leyva-Ramos et al., 2009; Morales-Saldana et al., 2007), or whose goal is to obtain a fixed-frequency PWM-based sliding mode controller (Chincholkar and Chan, 2017). Nevertheless, in this work the authors use a reduced number of state variables, specifically they only use one current for its implementation instead of two. Finally, related with robust control solutions, there is Alonge et al. (2015), where the authors consider the framework of Hammerstein's model for proposing a robust controller. Indeed, a set of fourth-order transfer functions are identified with the Hammerstein's approach, and the set of identified transfer functions are used to design a suited robust control technique that allows to deal with converter parameter uncertainties and load variations.

All these mentioned works, except few cases (Chen et al., 2016; Lopez-Santos et al., 2015), are based on a constant switching frequency, changing the duty cycle for tracking the voltage reference. Moreover, the design of the controller is based on a small-signal model obtained from the averaged statespace system. This means that the design specifications hold only in a neighborhood of the equilibrium point. For these reasons, theories about hybrid dynamical systems present a suited framework for modeling switching power systems, since it is possible to capture both continuous- and discrete-time dynamics and combine them in a unique model, without approximations. Moreover, the constraint about a fixed switching frequency is not more strictly necessary in this kind of context. Some preliminary results about the application of these hybrid theory to buck-boost converters are presented in Iannelli et al. (2008); Mariethoz et al. (2010). Likewise, Albea-Sanchez et al. (2015); Sferlazza et al. (2019) propose control solutions for DC-DC converters in which stability guarantees are established using recent tools from hybrid dynamical systems theory. Moreover, Sanchez et al. (2019) provides practical stabilization of operating points for switched affine systems, ensuring a minimum dwell-time associated with an LQR performance level during the transient response and an admissible chattering around the operating point. Using the same framework, in Albea Sanchez et al. (2019) a similar controller is proposed, but for a half-bridge inverter.

This work is inspired from Albea-Sanchez et al. (2015), for controlling a $\mathrm{QBC}$, whose topology, from the electronic architecture point of view, is more complex than the standard boost converters selected in Albea-Sanchez et al. (2015). In particular, the advantage of this QBC, with respect to a classical boost converter, is the higher steady-state gain (two times higher, approximately). Indeed, to obtain the same steady-state gain with a boost converter, one has to implement two converters con- nected in cascade, which requires two switching, increasing the commutation losses. In the $\mathrm{QBC}$, one can obtain the same gain using only one conversion stage increasing the efficiency.

The novelty of this paper is to integrate a state observer in a hybrid control scheme of the converter to avoid using current sensors. To the best of Authors' knowledge, an observer has not been designed before for a switched affine system controlled with a Lyapunov matrix-based min-projection control, in a hybrid dynamical model. This fact is particularly important because it allows to remove current sensors. From a practical point of view, the lack of current sensors can improve the production cost of converters. Indeed, if currents are not measured, it is not needed to employ neither current sensors, nor Analog to Digital Converters (ADC) any more; and the dimensions of the board could be better optimized. Moreover, the fact of using a QBC, which is four dimensions, justifies the use of an observer to reduce the number of sensors. The stability of the complete system is established in terms of Uniform Global Asymptotic Stability (UGAS) of a bounded and closed set that includes the operating point, providing UGAS of both tracking error of the equilibrium point and estimation error. It is useful to point out that the property of UGAS of such set implies the robustness of the complete control loop with respect to small perturbations around the operating point.

Finally, experimental results show the effectiveness of the proposed method. Indeed, the proposed hybrid control technique is compared with a PI controller, which is designed from an averaged model in continuous time, showing that the proposed strategy presents two main advantages in transient times. The first one is a direct control of the voltage and current trajectories, allowing to obtain no-inrush current at start-up, as well as, a better tracking of the voltage reference. The second advantage is the possibility of choosing the trajectory by tuning a pre-defined parameter, which allows to act directly in the number of switching, and consequently to act on the ripple of the inductor current.

The rest of the paper is organized as follows. The QBC model and the problem statement are described in Section 2. An observer for the unmeasured variable, as well as a hybrid control strategy are designed in Section 3 and 4 respectively. Experimental results are presented in Section 5. The paper closes with a section of conclusions.

Notation: Through out the paper $\mathbb{R}$ denotes the set of real numbers, $\mathbb{R}^{n}$ the $\mathrm{n}$-dimensional euclidean space and $\mathbb{R}^{n \times m}$ the set of all real $n \times m$ matrices. $M>0$ (resp. $M \prec 0$ ) represents that $M$ is a symmetric positive (resp. negative) definite matrix. The symbol "^" connotes the estimated variables. The Euclidan norm of vector $x \in \mathbb{R}$ is denoted by $\|x\| . \quad \lambda_{m}(M)$ and $\lambda_{M}(M)$ represent the minimum and maximum eigenvalues of $M$.

\section{Mathematical model and problem formulation}

The electric scheme of the QBC under analysis is depicted in Fig. 1. It exhibits the presence of two inductors $\left(L_{1}, L_{2}\right)$, two capacitors $\left(C_{1}, C_{2}\right)$, four diodes $\left(D_{1}, D_{2}, D_{3}, D_{4}\right)$ and one electronic switch $\left(S_{1}\right)$. The parasitic resistances of the inductors $\left(r_{L_{1}}, r_{L_{2}}\right)$ and of the capacitors $\left(r_{C_{1}}, r_{C_{2}}\right)$ are also considered. 
The mathematical model of this QBC can be expressed as a switched system in a state-space formulation, using the formalism given in Liberzon (2012). In particular, under the assumption that the converter works in Continuous Conduction Mode (CCM), two operation modes can be considered, called, respectively, mode $O N$ and mode $O F F$. The converter works in mode $O N$ when the electronic switch, $S_{1}$, is turned on, otherwise it works in mode $O F F$. For this reason, by applying the Kirchhofs's law to the circuit in Fig. 1, it is possible to write the following equations

$$
\left\{\begin{array}{l}
\dot{x}=A_{u} x+B \\
y=C_{u} x
\end{array}\right.
$$

where $x=\left[\begin{array}{llll}i_{L_{1}} & i_{L_{2}} & v_{C_{1}} & v_{C_{2}}\end{array}\right]^{\top}$ is the state vector, $u \in\{0,1\}$ is the switched control input, such that, $u=1$ means that the switch $S_{1}$ is turned on, while $u=0$ means that the switch $S_{1}$ is turned off, and $y=V_{O U T}$ is the output voltage. The matrices of the model (1) are

$$
\begin{aligned}
& A_{0}=\left[\begin{array}{ccrc}
-\frac{r_{L_{1}} R_{t}+r_{C_{2}} R_{t_{1}}}{L_{1} R_{t}} & \frac{r_{C_{1}} r_{C_{2}}}{L_{1} R_{t}} & \frac{r_{C_{2}}}{L_{1} R_{t}} & -\frac{R_{t_{1}}}{L_{1} R_{t}} \\
\frac{r_{C_{1}} r_{C_{2}}}{L_{2} R_{t}} & -\frac{r_{L_{2}} R_{t}+r_{C_{1}} R_{t_{2}}}{L_{2} R_{t}} & -\frac{R_{t_{2}}}{L_{2} R_{t}} & \frac{r_{C_{1}}}{L_{2} R_{t}} \\
-\frac{r_{C_{2}}}{C_{1} R_{t}} & \frac{R_{t_{2}}}{C_{1} R_{t}} & -\frac{1}{C_{1} R_{t}} & -\frac{1}{C_{1} R_{t}} \\
\frac{R_{t_{1}}}{C_{2} R_{t}} & -\frac{r_{C_{1}}}{C_{2} R_{t}} & -\frac{1}{C_{2} R_{t}} & -\frac{1}{C_{2} R_{t}}
\end{array}\right], \\
& A_{1}=\left[\begin{array}{cccc}
-\frac{r_{L_{1}}}{L_{1}} & 0 & 0 & 0 \\
0 & -\frac{r_{L_{2}} R_{t}+r_{C_{2}} R_{t_{1}}}{L_{C_{1}} R_{t}} & -\frac{r_{C_{2}}}{L_{2} R_{t}} & \frac{R_{t_{1}}}{L_{2} R_{t}} \\
0 & \frac{r_{C_{2}}}{C_{1} R_{t}} & -\frac{1}{C_{1} R_{t}} & -\frac{1}{C_{1} R_{t}} \\
0 & -\frac{R_{t_{1}}}{C_{2} R_{t}} & -\frac{1}{C_{2} R_{t}} & -\frac{1}{C_{2} R_{t}}
\end{array}\right], \\
& B=\left[\begin{array}{c}
\frac{V_{I N}}{L_{1}} \\
0 \\
0 \\
0
\end{array}\right], \quad C_{0}=\left[\begin{array}{c}
\frac{R_{0} r_{C_{1}}}{R_{t}} \\
\frac{R_{0} r_{1}}{R_{t}} \\
\frac{R_{0}}{R_{t}} \\
\frac{R_{0}}{R_{t}}
\end{array}\right]^{\top}, C_{1}=\left[\begin{array}{c}
0 \\
\frac{R_{0} r_{C_{2}}}{R_{t}} \\
\frac{R_{0}}{R_{t}} \\
\frac{R_{0}}{R_{t}}
\end{array}\right]^{\top} .
\end{aligned}
$$

where $V_{I N}$ is the input voltage, $R_{t}=R_{0}+r_{C_{1}}+r_{C_{2}}, R_{t_{1}}=R_{0}+r_{C_{1}}$ and $R_{t_{2}}=R_{0}+r_{C_{2}}$.

As mentioned in the Introduction, the most of the works in the literature do not use model (1) for control design purposes, but a State-Space Averaged (SSA) model. The SSA method presents some advantages, but also some drawbacks.

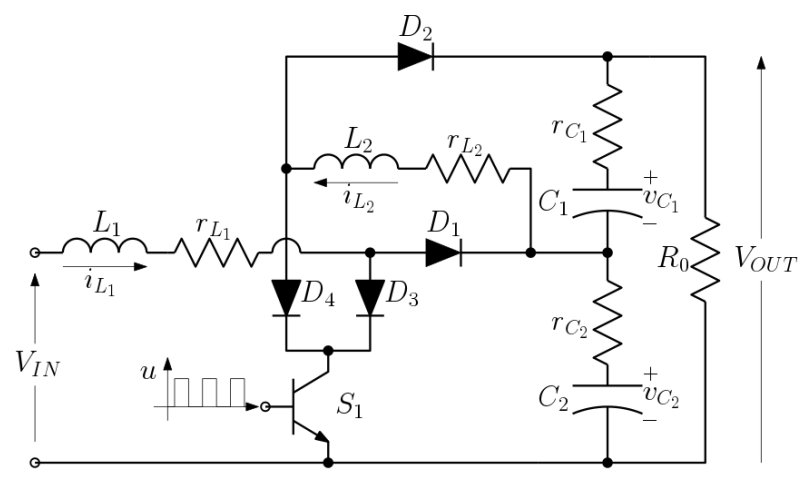

Figure 1: Electrical scheme of the QBC.

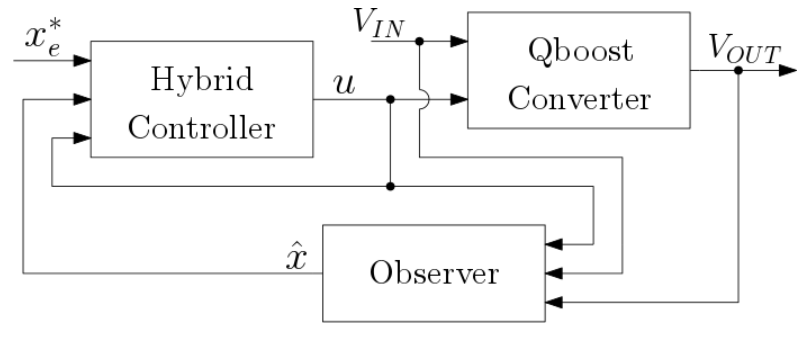

Figure 2: Block diagram of the proposed controlled system.

The main advantage is the simplicity of the control design. Indeed, the control law can be designed considering a standard linear time-invariant model. However, the drawbacks of this approach are mainly two. The first one, the design is based on a small-signal model, obtained by linearization around the working point. This means that if a wide range of operation is considered, the behaviors of the controlled system could not be appropriated. The second drawback is the constrained structure of the controller, which forces the signal to be updated periodically with a fixed switching frequency. This could facilitate the design of the converter (i.e. the choice of inductances, capacitors, and so on), but it removes a degree of freedom that could be used to minimize the number of commutations, and consequently the energy losses. Conversely, in this work, a different paradigm for approaching the control of the proposed QBC is given, by explicitly considering the switching behavior of the system and by relaxing the constraint of constant switching frequency.

\subsection{Problem Formulation}

The aim of this work is to design a hybrid control scheme for ensuring suitable convergence properties of the plant state, $x$, to a certain operating point, $x_{e}$. The specific objectives are:

- to obtain the operating point $x_{e}^{*}$, which represents the reference state, related with the desired voltage output, $V_{O U T}^{*}$.

- To design an observer that provides an estimation, $\hat{x}$, of the state, $x$, of the system, based on the input-output voltage measurements, on the input $u$ and on the model of the system (1). The observer has to be designed in order to guarantee the convergence to zero of the estimation error.

- To design a controller, that guarantees the asymptotic stability of the system ensuring the convergence of $x$ to $x_{e}^{*}$.

One of the main challenge of this work is to provide a controller without having access to the complete state and, considering a more realistic model (differently to the SSA model) that explicitly considers the switching behavior of the system, allowing to manage the switching frequency.

The block diagram of the proposed control scheme is shown in Fig. 2. 


\subsection{Working point selection}

As first step, the selection of the operating point $x_{e}^{*}$ is defined, which represents the reference state related with the desired output voltage, $V_{\text {OUT }}^{*}$.

Note that a generic equilibrium point $x_{e}$ is not necessarily an equilibrium point for the dynamics (1), but it can be an equilibrium for the switching system in a Filippov's generalization. In the case under study, the set of admissible equilibrium points, $\Omega_{e} \subset \mathbb{R}^{n}$, is defined as

$$
\begin{array}{r}
\Omega_{e}:=\left\{x_{e} \in \mathbb{R}^{n} \mid \text { there is } \lambda \in \Lambda \text { s.t. } \sum_{i=0}^{1} \lambda_{i}\left(A_{i} x_{e}+B\right)=0,\right. \\
\text { and } \left.\sum_{i=0}^{1} \lambda_{i} A_{i} \text { is Hurwitz }\right\},
\end{array}
$$

where

$$
\Lambda=\left\{\lambda \in[0,1]: \lambda_{0}+\lambda_{1}=1\right\} .
$$

Hence, an equilibrium point is admissible if it is an equilibrium point for the averaged dynamics

$$
\dot{x}=\sum_{i=0}^{1} \lambda_{i}\left(A_{i} x+B\right), \text { for }\left[\lambda_{0}, \lambda_{1}\right] \in \Lambda,
$$

and stability properties are guaranteed for the corresponding convex dynamics.

Obviously for the case under study the pairs $\left(\lambda_{0}, \lambda_{1}\right)$ and the associated equilibrium points $x_{e} \in \Omega_{e}$ are infinite. However, the goal is to regulate the output voltage to a fixed value $V_{O U T}$, which is related to a specific equilibrium point. Then, the following further constraint has to be considered,

$$
y=\sum_{i=0}^{1} \lambda_{i} C_{i} x_{e}=V_{O U T} .
$$

For these reasons the operating point $x_{e}^{*}$ is defined, with associated values $\left(\lambda_{0}^{*}, \lambda_{1}^{*}\right)$ such that $\lambda_{0}^{*}+\lambda_{1}^{*}=1$, as follows:

$$
x_{e}^{*}=-\left(\sum_{i=0}^{1} \lambda_{i}^{*} A_{i}\right)^{-1} B, \text { for } i \in\{0,1\},
$$

such that:

$$
V_{\text {OUT }}^{*}=\sum_{i=0}^{1} \lambda_{i}^{*} C_{i} x_{e}^{*}, \text { for } i \in\{0,1\} .
$$

In general, the solution can be founded analytically and/or numerically.

\section{Observer Design}

Since the complete system state is not measurable, it is necessary to provide an observer that estimates these quantities, and, particularly, the inductor currents.
Note that the system is not observable for all functioning modes. Indeed, when the switch is $O N$ mode, the observ-

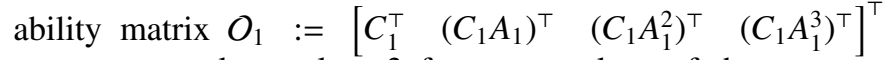
presents a rank equal to 3 for some values of the parameters. Therefore, the system is not observable in this mode. Nevertheless, the QBC is observable when it is working on $O F F$ mode because of the observability matrix $O_{0}:=$

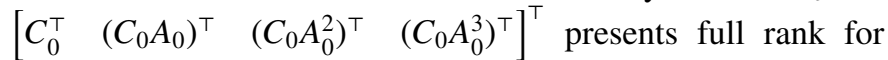
any values of the parameters. Note that this problem is the same one observed for the standard boost case (Liu et al., 2014), and it presents a physical interpretation. Indeed, it is easy to see in Fig. 1 that when the converter is working on $O N$ mode, there is not information about the current on the inductor, $L_{1}$, which is being inferred from the output.

Now, since the system periodically jumps between two modes, i.e. it never remains in $O N$ or $O F F$ mode for an undetermined amount of time, the idea is to design an observer based on (1), driven by the residual

$$
e_{y}=y-\hat{y}=V_{\text {OUT }}-C_{u} \hat{x}=C_{u} \tilde{x}_{o}, \quad \text { with } \quad \tilde{x}_{o}=x-\hat{x},
$$

only during the $O F F$ mode.

The proposed switching observer is

$$
\dot{\hat{x}}=A_{u} \hat{x}+B+(u-1) K_{f} C_{u} \tilde{x}_{o} .
$$

Note that when $u=1$, the corrective term is equal to zero and (8) becomes the original plant, while for $u=0$ the dynamics is driven by the output error (7).

For observer dynamics (8), the following result holds.

Lemma 1. If there exist matrices $P_{o} \in \mathbb{R}^{4 \times 4}>0$ and $Q_{o} \in$ $\mathbb{R}^{4 \times 4}>0$ and a matrix $L \in \mathbb{R}^{4 \times 1}$, such that, the following Linear Matrices Inequalities (LMIs) are satisfied,

$$
\begin{aligned}
P_{o} A_{1}+A_{1}^{\top} P_{o} & \prec-Q_{o} \\
P_{o} A_{0}+L C_{0}+A_{0}^{\top} P_{o}+C_{o} L^{\top} & \prec-Q_{o}
\end{aligned}
$$

then by choosing the observer gain $K_{f}=P_{o}^{-1} L, \hat{x}$ converges to $x$ asymptotically along the dynamics (1) and (8).

Proof. In order to proof the theorem statement, the switching systems theory given in Liberzon (2012) is used. In particular, the following common Lyapunov function

$$
V_{o}\left(\tilde{x}_{o}\right)=\frac{1}{2} \tilde{x}_{o}^{\top} P_{o} \tilde{x}_{o}
$$

is considered.

The derivative of (11) along the dynamics (1) and (8) gives

$$
\begin{aligned}
\dot{V}_{o}\left(\tilde{x}_{o}\right) & =\tilde{x}_{o}^{\top} P_{o} \dot{\tilde{x}}_{o}=\tilde{x}_{o}^{\top} P_{o}\left(A_{u}+(u-1) K_{f} C_{u}\right) \tilde{x}_{o} \\
& = \begin{cases}\tilde{x}_{o}^{\top} P_{o} A_{1} \tilde{x}_{o} & \text { if } u=1 \\
\tilde{x}_{o}^{\top} P_{o}\left(A_{0}+K_{f} C_{0}\right) \tilde{x}_{o} & \text { if } u=0\end{cases} \\
& \prec-\tilde{x}_{o}^{\top} Q_{o} \tilde{x}_{o} .
\end{aligned}
$$

It is worth noting that the two assumptions (9)-(10), as well as, the fact that $K_{f}=P_{o}^{-1} L$ are considered in (12). Moreover, 
since $\tilde{x}_{o}^{+}=\tilde{x}_{o}$, during jumps, i.e., there is not a jump map in the observer dynamics, then

$$
V_{o}\left(\tilde{x}_{o}^{+}\right)-V\left(\tilde{x}_{o}\right)=0 .
$$

Consequently, the statement comes directly from Liberzon (2012, Theorem 2.1).

\section{The control Law}

Before introducing the proposed control strategy, it is necessary to satisfy the following assumption.

Assumption 1. Consider a given $V_{O U T}^{*}$ and its associated pair $\left(\lambda_{0}^{*}, \lambda_{1}^{*}\right)$ resulted from Section 2.2 and assume that there exist matrices $P \in \mathbb{R}^{4 \times 4}>0$ and $Q \in \mathbb{R}^{4 \times 4}>0$ that satisfy

$$
A_{u}^{\top} P+P A_{u}+2 Q<0 . \quad u \in\{0,1\} .
$$

It is easy to see that if each $A_{u}$ is Hurwitz, then conditions (14) sre feasible.

Following the formalism given in Goebel et al. (2012), wherein the continuous-time behavior resembles the evolution in (1) and (8), and the discrete-time behavior captures the jump of the control signal $u$ from one mode to another one. The overall dynamics are represented as follows

$$
\mathcal{H}:=\left\{\begin{array}{l}
{\left[\begin{array}{c}
\dot{x} \\
\dot{u} \\
\dot{x} \\
\dot{\tau}
\end{array}\right]=f(x, u, \hat{x}, \tau), \quad(x, u, \hat{x}, \tau) \in C} \\
{\left[\begin{array}{l}
x^{+} \\
u^{+} \\
\hat{x}^{+} \\
\tau^{+}
\end{array}\right] \in \mathcal{G}(x, u, \hat{x}, \tau), \quad(x, u, \hat{x}, \tau) \in \mathcal{D},}
\end{array}\right.
$$

where

$$
f(x, u, \hat{x}, \tau)=\left[\begin{array}{c}
A_{u} x+B \\
0 \\
A_{u} \hat{x}+B+(u-1) K_{f} C_{u} \tilde{x}_{o} \\
1-\varsigma\left(\frac{\tau}{T}\right)
\end{array}\right],
$$

such that, the scalar $T>0$ and the dead-zone function defined as $\varsigma(s):=\max \{0, s-1\}, \forall s \geq 0$, denotes the flow map. Moreover,

$$
\mathcal{G}(x, u, \hat{x}, \tau)=\left[\begin{array}{c}
x \\
\arg \min _{u \in\{0,1\}}\left(\hat{x}-x_{e}^{*}\right)^{\top} P f_{3}(x, u, \hat{x}) \\
\hat{x} \\
0
\end{array}\right],
$$

where $f_{3}(x, u, \hat{x})=A_{u} \hat{x}+B+(u-1) K_{f} C_{u} \tilde{x}_{o}$ denotes a set-valued map capturing the switching logic, and $C$ and $\mathcal{D}$ represent the flow and jump sets, respectively, being defined as

$$
\begin{aligned}
C:= & \left\{x, u, \hat{x}: \tilde{x}^{\top} \bar{P} \dot{\tilde{x}} \leq-\eta \tilde{x}^{\top} \bar{Q} \tilde{x}\right\} \times[0,2 T] \\
& \cup\{x, u, \hat{x}, \tau: \tau \in[0, T]\}, \\
\mathcal{D}:= & \left\{x, u, \hat{x}: \tilde{x}^{\top} \bar{P} \dot{\tilde{x}} \geq-\eta \tilde{x}^{\top} \bar{Q} \tilde{x}\right\} \times[T, 2 T],
\end{aligned}
$$

where $\tilde{x}=\left[\begin{array}{c}\tilde{x}_{s} \\ \tilde{x}_{o}\end{array}\right]$, with $\tilde{x}_{s}=\hat{x}-x_{e}^{*}$, is the vector error containing both the state and the estimation error, such that,

$$
\dot{\tilde{x}}=\bar{A}_{u} \tilde{x}+\bar{B},
$$

with $\bar{A}_{u}:=\left[\begin{array}{c|c}A_{u} & (u-1) K_{f} C_{u} \\ \hline 0 & A_{u}+(u-1) K_{f} C_{u}\end{array}\right]$ and $\bar{B}:=\left[\begin{array}{c}B+A_{u} x_{e} \\ 0\end{array}\right]$. Finally, matrices $\bar{P}$ and $\bar{Q}$ are defined as follows

$$
\begin{gathered}
\bar{P}:=\left[\begin{array}{c|c}
P & 0 \\
\hline 0 & P_{o}
\end{array}\right], \\
\bar{Q}:=\left[\begin{array}{c|c}
Q & Q_{o} \\
\hline 0 & Q_{o}
\end{array}\right],
\end{gathered}
$$

and $\eta \in(0,1)$ is a design parameter chosen to achieve a tradeoff between switching frequency and a LQR-optimization level, as given in Albea-Sanchez et al. (2015, Theorem 2).

Note that the dead-zone function $\varsigma\left(\frac{\tau}{T}\right)$ allows to ensure that all maximal solutions are complete. Then, using the hybrid dynamical theory given in Goebel et al. (2012), stability property of $\tilde{x}$ is guaranteed. Indeed, UGAS of the compact attractor:

$$
\mathcal{A}:=\{(x, u, \hat{x}):\|\tilde{x}\| \leq X, u \in\{0,1\}, \tau \in[0,2 T]\}
$$

is established.

The following lemmas are considered, before giving the main result.

Lemma 2. Consider $a V_{O U T}^{*}$ and its associated working point $x_{e}^{*}$ and pair $\left(\lambda_{0}^{*}, \lambda_{1}^{*}\right)$ selected from Eq.s (5)-(6). Moreover, consider $P>0$ and $Q>0$ of suited dimension satisfying Assumption 1 , and $P_{o}>0, Q_{o}>0$ and $K_{f}$ such that, conditions (9)(10) are satisfied. Then, for each $\hat{x}$,

$$
\min _{u \in\{0,1\}} \tilde{x}^{\top} \bar{P}\left[\begin{array}{c}
A_{u} \hat{x}+B+(u-1) K_{f} C_{u} \tilde{x}_{o} \\
\left(A_{u}+(u-1) K_{f} C_{u}\right) \tilde{x}_{o}
\end{array}\right] \leq-\tilde{x}^{\top} \bar{Q} \tilde{x} .
$$

Proof. Considering that (5) is linear in $\lambda_{u}$, and $\lambda_{u}$ belongs to the compact set (3), then the following minimum can be computed at the extreme points,

$$
\begin{aligned}
& \min _{u \in\{0,1\}} \tilde{x}^{\top} \bar{P}\left[\begin{array}{c}
A_{u} \hat{x}+B+(u-1) K_{f} C_{u} \tilde{x}_{o} \\
\left(A_{u}+(u-1) K_{f} C_{u}\right) \tilde{x}_{o}
\end{array}\right] \\
& =\min _{\lambda_{u} \in \Lambda} \tilde{x}^{\top} \bar{P}\left[\begin{array}{c}
\left(\sum_{u=0}^{1} \lambda_{u}^{*} A_{u}\right) x_{e}^{*}+B \\
0
\end{array}\right] \\
& \leq \min _{\lambda_{u} \in \Lambda} \tilde{x}^{\top} \bar{P}\left[\begin{array}{c}
\left(\sum_{u=0}^{1} \lambda_{u}^{*} A_{u}\right) x_{e}^{*}+B \\
0
\end{array}\right]=0 .
\end{aligned}
$$

Then, from (14), (9) and (10),

$$
\begin{aligned}
& \min _{u \in\{0,1\}} \tilde{x}^{\top} \bar{P}\left[\begin{array}{c}
A_{u} \hat{x}+B+(u-1) K_{f} C_{u} \tilde{x}_{o} \\
\left(A_{u}+(u-1) K_{f} C_{u}\right) \tilde{x}_{o}
\end{array}\right] \\
& =\min _{\lambda_{u} \in \Lambda} \tilde{x}^{\top} \bar{P}\left[\begin{array}{c}
A_{u}\left(\tilde{x}_{s}+x_{e}^{*}\right)+B+(u-1) K_{f} C_{u} \tilde{x}_{o} \\
\left(A_{u}+(u-1) K_{f} C_{u}\right) \tilde{x}_{o}
\end{array}\right] \\
& =\min _{\lambda_{u} \in \Lambda} \tilde{x}^{\top} \bar{P}\left[\begin{array}{c}
A_{u} \tilde{x}_{s}+(u-1) K_{f} C_{u} \tilde{x}_{o} \\
\left(A_{u}+(u-1) K_{f} C_{u}\right) \tilde{x}_{o}
\end{array}\right] \\
& \leq-\tilde{x}^{\top} \bar{Q} \tilde{x},
\end{aligned}
$$


where the second equality comes from $A_{u} x_{e}^{*}+B=0$ for $u \in$ $\{0,1\}$ and the last inequality is derived from (14) and Lemma 1.

Lemma 3. ( Albea Sanchez et al., 2019, Lemma 2) All the eigenvalues of the matrix $\bar{P}^{-1} \bar{Q}$ are positive and $\left\|e^{\bar{A}_{u} t}\right\| \leq$ $\frac{\lambda_{M}^{1 / 2}(\bar{P})}{\lambda_{m}^{1 / 2}(\bar{P})} e^{-\alpha t}$, where $\alpha=\lambda_{m}\left(\bar{P}^{-1} \bar{Q}\right)$.

Now, a practical minimum dwell-time property for $\mathcal{H}$ is established.

Property 1. There exists a scalar $T^{*}>0$, such that, for any chosen $T \leq T^{*}$ the solutions to hybrid system $\mathcal{H}$ flow for at least $T$ ordinary time after the jump, before reaching set $\mathcal{D}$.

Proof. It is considered that in $t=t_{0}$ occurs the first jump, letting define $\tilde{t}:=t-t_{0}$. Then, the trajectories of the dynamics (18) flowing in $C$, are defined as follows,

$$
\tilde{x}=e^{\bar{A}_{u} \tilde{t}}\left(\tilde{x}_{0}+\bar{A}_{u}^{-1} \bar{B}\right)-\bar{A}_{u}^{-1} \bar{B}
$$

From the norm properties and applying Lemma 3, it results

$$
\left\|\tilde{x}(\tilde{t})-\bar{A}_{u}^{-1} \bar{B}\right\| \leq \frac{\lambda_{M}^{1 / 2}(\bar{P})}{\lambda_{m}^{1 / 2}(\bar{P})} e^{\alpha \tilde{t}}\left\|\tilde{x}_{0}+\bar{A}_{u}^{-1} \bar{B}\right\| .
$$

Then, for all $0 \leq \tilde{t} \leq T$, it follows

$$
0 \leq \tilde{t} \leq \frac{1}{\alpha} \operatorname{Ln}\left(\frac{\lambda_{M}^{1 / 2}(\bar{P})\left\|\tilde{x}_{0}+\bar{A}_{u}^{-1} \bar{B}\right\|}{\lambda_{m}^{1 / 2}(\bar{P})\left\|\tilde{x}(\tilde{t})-\bar{A}_{u}^{-1} \bar{B}\right\|}\right)
$$

Note that the solutions are in $\mathcal{D}$, at time $\tilde{t}=T$. Therefore, dwell-time upper bound, $T^{*} \geq T \geq 0$, can be defined as

$$
T^{*}=\frac{1}{\alpha} \operatorname{Ln}\left(\frac{\lambda_{M}^{1 / 2}(\bar{P})\left\|\tilde{x}_{0}+\bar{A}_{u}^{-1} \bar{B}\right\|}{\lambda_{m}^{1 / 2}(\bar{P})\left\|\tilde{x}\left(T^{*}\right)-\bar{A}_{u}^{-1} \bar{B}\right\|}\right) .
$$

Hence, the maximum chattering induced by $T^{*}$ is $\tilde{x}_{0}$ and $\tilde{x}\left(T^{*}\right)$. Now, the main result can be given.

Theorem 1. Consider $a V_{O U T}^{*}$ and its associated working point $x_{e}^{*}$ and pair $\left(\lambda_{0}^{*}, \lambda_{1}^{*}\right)$ selected from Eq.s (5)-(6). Moreover, consider $x_{e}^{*} \in \mathbb{R}^{4 \times 1}$, matrices $P \in \mathbb{R}^{4 \times 4}>0$ and $Q \in \mathbb{R}^{4}>0$ satisfying Assumption 1, and matrices $P_{o} \in \mathbb{R}^{4 \times 4}>0, Q_{o} \in \mathbb{R}^{4 \times 4}>0$ and vector $K_{f} \in \mathbb{R}^{4 \times 1}$ selected such that Lemma 1 satisfies conditions (9)-(10). Then, for any scalar $0 \leq T \leq T^{*}$ the following holds:

\section{1. set $\mathcal{A}$ is compact,}

2. set $\mathcal{A}$ is $U G A S$ for hybrid system (15),

3. set $\mathcal{A}_{0}:=\{(x, u, \hat{x}): \tilde{x}=\{0\}, u \in\{0,1\}, \tau \in[0,2 T]\}$ is globally practically asymptotically stable for system (15). As long as $T$ is small enough, set $\mathcal{A}$ can be arbitrarily close to $\mathcal{A}_{0}$.
Proof. From the properties of $f, \mathcal{G}, C$ and $\mathcal{D}$, system $\mathcal{H}$ satisfies the hybrid basic conditions of Goebel et al. (2012, Ass. 6.5), and thus, useful well-posed hybrid results can be applied.

Proof of item 1) Hybrid system (15) satisfies Property 1, then a bound of $\tilde{x}$ in $C$ can be found as follows

$$
\|\tilde{x}(t)\| \leq \frac{\lambda_{M}^{1 / 2}(\bar{P})}{\lambda_{m}^{1 / 2}(\bar{P})} e^{\alpha \tilde{t}}\left\|\tilde{x}\left(t_{0}\right)+\bar{A}_{u}^{-1} \bar{B}\right\|+\left\|\bar{A}_{u}^{-1} \bar{B}\right\|:=X,
$$

considering $t_{0}$, a time instant where occurs any jump in the steady-state. Consequently, $\mathcal{A}$ is compact.

Proof of item 2) Consider the following Lyapunov function,

$$
V(\tilde{x})=\frac{1}{2} \tilde{x}^{\top} \bar{P} \tilde{x}
$$

During flows

$$
\begin{aligned}
& \langle\nabla V(\tilde{x}), f(x, u, \hat{x})\rangle=\tilde{x}^{\top} \bar{P}\left[\begin{array}{c}
A_{u} \hat{x}+B+(u-1) K_{f} C_{u} \tilde{x}_{o} \\
\left(A_{u}+(u-1) K_{f} C_{u}\right) \tilde{x}_{o}
\end{array}\right] \\
& \leq-\tilde{x}^{\top} \bar{Q} \tilde{x} .
\end{aligned}
$$

This fact comes directly from the flow set $C$ definition, (16), and Lemma 1 . When a jump occurs,

$$
V\left(\tilde{x}^{+}\right)-V(\tilde{x})=0
$$

since $\tilde{x}^{+}=\left[\begin{array}{c}\hat{x}^{+}-x_{e}^{*} \\ x^{+}-\hat{x}^{+}\end{array}\right]=\left[\begin{array}{c}\hat{x}-x_{e}^{*} \\ x-\hat{x}\end{array}\right]=\tilde{x}$.

UGAS of $\mathcal{A}$ is shown using Prieur et al. (2014, Theorem 1). In particular, since the distance of $x$ to the attractor (19) is defined by $\left|x-x_{e}^{*}\right|=|\tilde{x}|$, it results that Prieur et al. (2014, Equation (6)) is satisfied from the structure of the Lyapunov function (23) and from (24) and (25). Moreover, Prieur et al. (2014, Theorem 1) requires the construction of the restricted hybrid system $\mathcal{H}_{\delta, \Delta}$ by intersecting $C$ and $\mathcal{D}$ with the set,

$$
\mathcal{S}_{\delta, \Delta}=\{(\tilde{x}, u):|\tilde{x}| \geq \delta \text { and }|\tilde{x}| \leq \Delta\},
$$

and proving semi-global practical persistence flow for $\mathcal{H}_{\delta, \Delta}$, for each value of $(\delta, \Delta)$. In particular, in order to show the practical persistent flow, it needs to be proven that there exist $\gamma \in \mathcal{K}_{\infty}$ and the scalar $N \geq 0$, such that all solutions to $\mathcal{H}_{\delta, \Delta}$ satisfy,

$$
t \geq \gamma(j)-N, \quad \forall t \in \bigcup_{j \in \operatorname{dom}_{j} \xi} I^{j} \times\{j\}
$$

see Prieur et al. (2014) for details. To establish (27), it results that after each jump,

$$
\begin{aligned}
& \tilde{x}^{\top} \bar{P} \tilde{x}^{+}=\tilde{x}^{\top} \bar{P}\left[\begin{array}{c}
A_{u^{+}} \hat{x}+B+\left(u-1^{+}\right) K_{f} C_{u^{+}} \tilde{x}_{o} \\
\left(A_{u^{+}}+\left(u-1^{+}\right) K_{f} C_{u^{+}}\right) \tilde{x}_{o}
\end{array}\right] \\
&=\tilde{x}^{\top} \bar{P}\left[\begin{array}{c}
\min _{u \in\{0,1\}} A_{u^{+}} \hat{x}+B+\left(u-1^{+}\right) K_{f} C_{u^{+}} \tilde{x}_{o} \\
\left(A_{u^{+}}+\left(u-1^{+}\right) K_{f} C_{u^{+}}\right) \tilde{x}_{o}
\end{array}\right] \\
& \leq-\tilde{x}^{\top} \bar{Q} \tilde{x}<-\eta \tilde{x}^{\top} \bar{Q} \tilde{x},
\end{aligned}
$$

from inequalities (9)-(10), and Lemma 2. Moreover, the strictly inequality is due to the fact that $\eta<1$. Therefore, if any solution to $\mathcal{H}_{\delta, \Delta}$ performs a jump from $\mathcal{S}_{\delta, \Delta}$ it remains in $\mathcal{S}_{\delta, \Delta}$ because $\hat{x}^{+}=\hat{x}$ and $x^{+}=x$, thus, $\tilde{x}$ does not change. Then, from 


\begin{tabular}{lll}
\multicolumn{3}{c}{ Table 1: Circuit parameters values } \\
CoMPONENT & VALUE & ModeL \\
\hline$V_{I N}$ & $30 \mathrm{~V}$ & \\
$L_{1}$ & $330 \mu \mathrm{H}$ & AGP4233-334ME \\
$L_{2}$ & $470 \mu \mathrm{H}$ & AGP4233-474ME \\
$r_{L_{1}}, r_{L_{1}}$ & $11.5 \mathrm{~m} \Omega$ & \\
$C_{1}, C_{2}$ & $20 \mu \mathrm{F}$ & MKP1848C62090JP4 \\
$r_{C_{1}}, r_{C_{1}}$ & $5 \mathrm{~m} \Omega$ & \\
$R_{0}$ & $390 \Omega$ & \\
$D_{1,2,3,4}$ & & C3D06060A \\
$S_{1}$ & & C3M0065090D \\
Driver & & 1EDI20N12AF \\
\hline
\end{tabular}

(17), it jumps to the interior of the flow set $C \cap \mathcal{S}_{\delta, \Delta}$. Likewise, from strict inequality in (28), all non-terminating solutions must flow for some time and since $C \cap \mathcal{S}_{\delta, \Delta}$ is bounded, there is a uniform dwell-time $\rho(\delta, \Delta)$ between each pair of consecutive jumps. This dwell-time $(\delta, \Delta)$ implies Prieur et al. (2014, Equation (4)) with the class- $\mathcal{K}_{\infty}$ function $\gamma(j)=\rho(\delta, \Delta) j$ and $N=1$. Then, all assumptions of Prieur et al. (2014, Theorem 1) are satisfied and UGAS of $\mathcal{A}$ is concluded.

Proof of item 3) From Property 1, as $T$ tends to zero, the minimum dwell time tends also to zero. Then, set $\mathcal{A}$ shrinks to $\mathcal{A}_{0}$ from a sufficiently small $T$.

\section{Experimental validations}

\subsection{Experimental setup}

A test setup was built to validate the proposed hybrid control technique. The complete system is implemented as shown in the block diagram of Fig. 2, where the observer is described by Equation (8), and the hybrid controller selects the control variable $u$ according to the dynamics shown in (15). The employed test setup consists of:

- a QBC, whose topology is shown in Fig. 1 and the electrical parameters are given in Table 1.

- An electronic card with current sensors ${ }^{1}$ (LEM LTS 15NP) for the measurements of the inductor currents, and voltage sensors ${ }^{1}$ for the measurement of the capacitor voltages. Voltage sensors have been built by means of resistor dividers connected with operational amplifiers in buffer configuration.

- A dSPACE card (DS1103) with a PowerPC 604e at 400 $\mathrm{MHz}$ and a fixed-point DSP TMS320F240.

The system was implemented in a dSPACE ${ }^{\circledR}$ card by means of Matlab-Simulink ${ }^{\circledR}$. Both the QBC and the measurement card were embedded in the electronic card shown in Fig. 3.

\footnotetext{
${ }^{1}$ Current and voltage sensors are used to show the effectiveness of the observer, namely to show only that the estimated variable converge to the measured ones, only the output voltage measurement is used to preform the feedback.
}

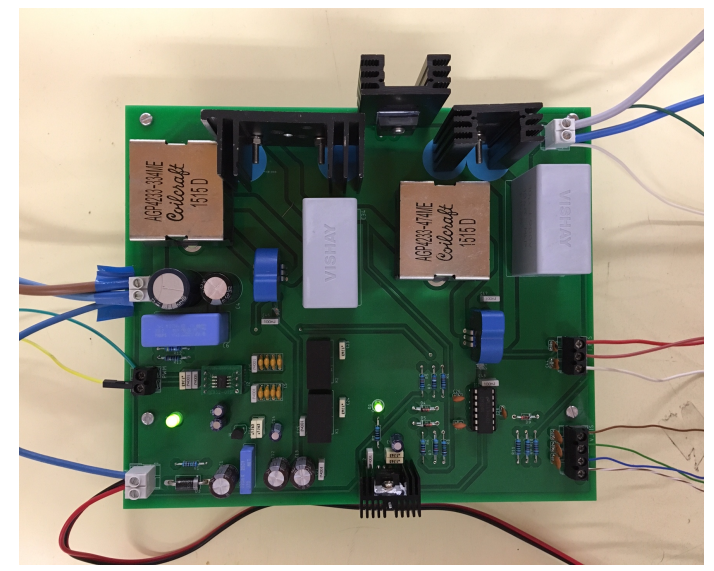

Figure 3: QBC prototype.

\subsection{Experimental results}

The following results were performed by selecting a desired output voltage $V_{O U T}^{*}=330 \mathrm{~V}$. For this value of $V_{O U T}^{*}$ the corre-

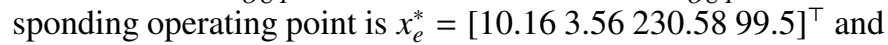
their associated $\lambda_{0}^{*}=0.292$ and $\lambda_{1}^{*}=0.708$. Moreover, $\eta=0.5$ is chosen from Albea-Sanchez et al. (2015, Theorem 2) and from the selected $\tilde{x}(\tilde{t})=\left[\begin{array}{llllllll}1 & 1 & 2 & 2 & 0.2 & 0.2 & 1 & 1\end{array}\right]^{\top} . T^{*}=0.001589$ was computed from Property 1 , considering a current ripple equal to $2.5 \mathrm{~A}$ for both $i_{L_{1}}$ and $i_{L_{2}}$ and a voltage ripple equal to $10 \mathrm{~V}$ for both $v_{C_{1}}$ and $v_{C_{2}}$. Moreover, $T$ was selected equal to $T=5 \mu \mathrm{s}$. Then, satisfying Assumption 1, they are obtained:

$$
P=\left[\begin{array}{cccc}
16.356 & -0.085 & -0.003 & 0.004 \\
-0.085 & 23.396 & 0.011 & -0.006 \\
-0.003 & 0.011 & 1.002 & 0.001 \\
0.004 & -0.006 & 0.001 & 1.000
\end{array}\right], Q=\left[\begin{array}{cccc}
16.194 & 5.273 & -12.211 & -8.957 \\
5.273 & 57.393 & -33.235 & -16.319 \\
-12.211 & -33.235 & 31.816 & 18.652 \\
-8.957 & -16.319 & 18.652 & 13.328
\end{array}\right]
$$

Finally, the values of $P_{o}, Q_{o}$ satisfying Lemma 1, that allow to compute the observer gain $K_{f}$, are

$$
P_{o}=\left[\begin{array}{cccc}
258.194 & -0.040 & 0.109 & 0.097 \\
-0.040 & 367.748 & 0.188 & -0.182 \\
0.109 & 0.188 & 15.652 & 0.000 \\
0.097 & -0.182 & 0.000 & 15.649
\end{array}\right], Q_{o}=10^{4}\left[\begin{array}{cccc}
1.139 & 0.334 & 0.002 & 0.005 \\
0.334 & 0.301 & 0.001 & 0.001 \\
0.002 & 0.001 & 0.165 & 0.151 \\
0.005 & 0.001 & 0.151 & 0.192
\end{array}\right],
$$

and $K_{f}$ is

$$
K_{f}=\left[\begin{array}{llll}
-0.354 & -0.409 & 32.436 & 17.085
\end{array}\right]^{\top} .
$$

Figure 4 shows the evolution of the output voltage in the start-up, applying the proposed control strategy given in (15). Note that the output converges to the desired $V_{\text {OUT }}^{*}$. Figures 5 and 6 show the inductor current evolutions, $i_{L_{1}}$ and $i_{L_{2}}$, also during the start-up test. Since the feedback is computed from the estimated variables, both measured and estimated variables are shown, as well as, their estimation errors. From these figures, no inrush currents occur, and after a short transient-time the estimated currents track the corresponding measured ones. Finally, both capacitor voltages $v_{C_{1}}$ and $v_{C_{2}}$ are given in Fig.s 7 and 8 . In these plots it is also shown that the observer variables converge to $v_{C_{1}}$ and $v_{C_{2}}$ with a small steady-state error.

In order to highlight some steady-state behaviors of the converter, Fig.s 9-13 show the output voltage evolution, inductor currents and capacitor voltages, at this time period. From Fig. 10 and 11 , it is noted an average value of $i_{L_{1}}$ and $i_{L_{2}}$ around 
their desired operating point given by $x_{e}^{*}$, and a current ripple $\Delta i_{L_{1}}=0.5$ and $\Delta i_{L_{2}}=1.5$, which are in the interior of the compact set $\mathcal{A}$, computed from $\tilde{x}(\tilde{t})$. In these figures, it is noted the effect of the "non-fixed" switching frequency originated from the proposed hybrid control strategy. Moreover, it is possible to reduce the ripple by decreasing the dwell time $T_{w}$ (at steady state). Experimentally, one can note that the switching frequency, in the steady-state, is about $1 /\left(2 \alpha T_{w}\right)$, where $\alpha \in\left[\begin{array}{ll}1 & 1.5\end{array}\right]$, and this frequency is maintained almost constant despite the equilibrium point changes. For example, $T_{w}=3 \mu \mathrm{s}$ results in a switching frequency of about $150 \mathrm{kHz}$ regardless of the equilibrium point. Hence, the above given empirical rule can be used for sizing the passive components such as EMI filters, reactive components, etc.

Therefore, the proposed approach is in a clear-cut contrast with conventional hysteresis-based controllers, where the switching frequency, at steady-state, depends on the load resistance and input voltage. Thus, the proposed strategy combines both a fast transient response of a hysteresis controller with variable switching frequency, and a predictable PWM behavior, at steady-state, with constant switching frequency.

From Fig.s 9-13, it is evident the capability of the observer to estimate the state variables with a small steady-state error.

The robustness of the proposed hybrid control strategy with respect to parameter variations can be pointed out by adding an outer loop for regulating the output voltage, as in LopezSantos et al. (2015). Fig. 14 depicts the converter response to a step variation in the load resistance from $390 \Omega$ to $250 \Omega$. Fig.s 15-16 show estimated and measured currents $i_{L_{1}}$ and $i_{L_{2}}$, as well as their estimation errors, while Fig.s 17-18 show estimated and measured voltages $v_{C_{1}}$ and $v_{C_{2}}$, as well as their estimation errors, during the above mentioned load variation. The fast response of the output voltage can be observed from Fig. 14, which shows the suited behavior provided by the proposed control strategy. Moreover, Fig.s 15-18 show the good tracking of the state variable provided by the observer, which is able to estimate currents $i_{L_{1}}$ and $i_{L_{2}}$, and voltages $v_{C_{1}}$ and $v_{C_{2}}$ with a small error, showing the effectiveness of the proposed estimation strategy. Finally, Fig. 19 shows the converter response to a step variation in the load resistance from $250 \Omega$ to $390 \Omega$, respectively. Also in this case same considerations given above can be inferred.

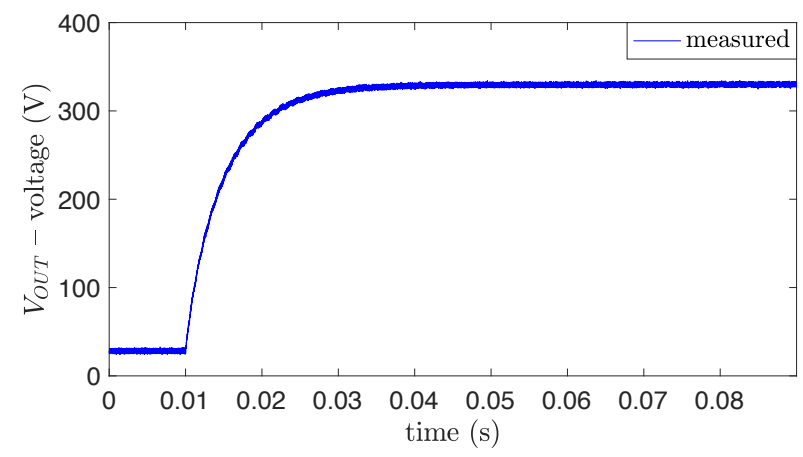

Figure 4: Output voltage during a start-up test.
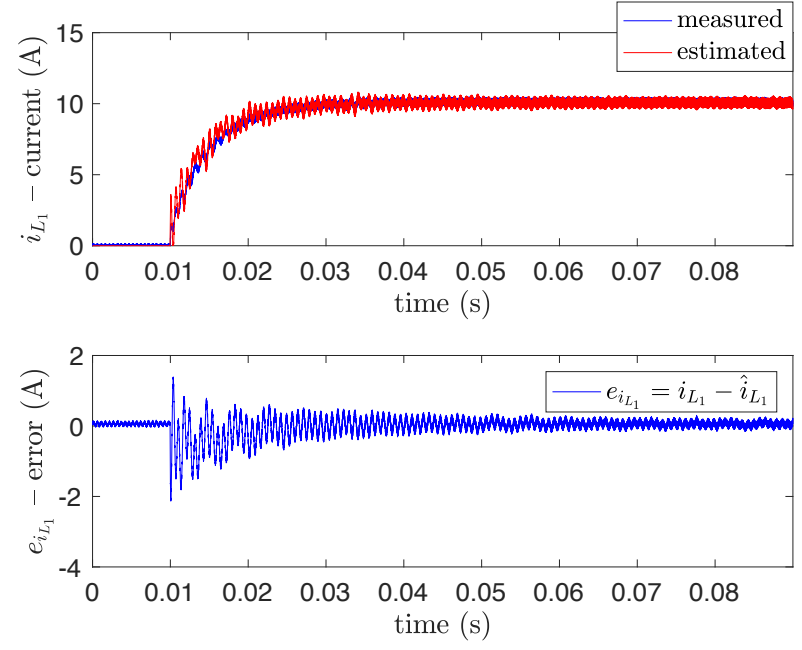

Figure 5: Estimated and measured current $i_{L_{1}}$, and its estimation error, during a start-up test.
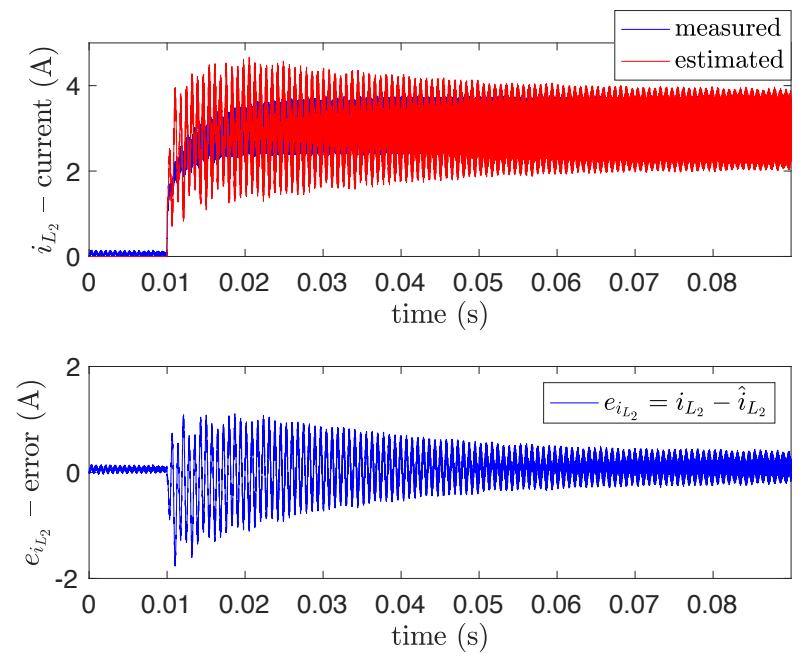

Figure 6: Estimated and measured current $i_{L_{2}}$, and its estimation error, during a start-up test.
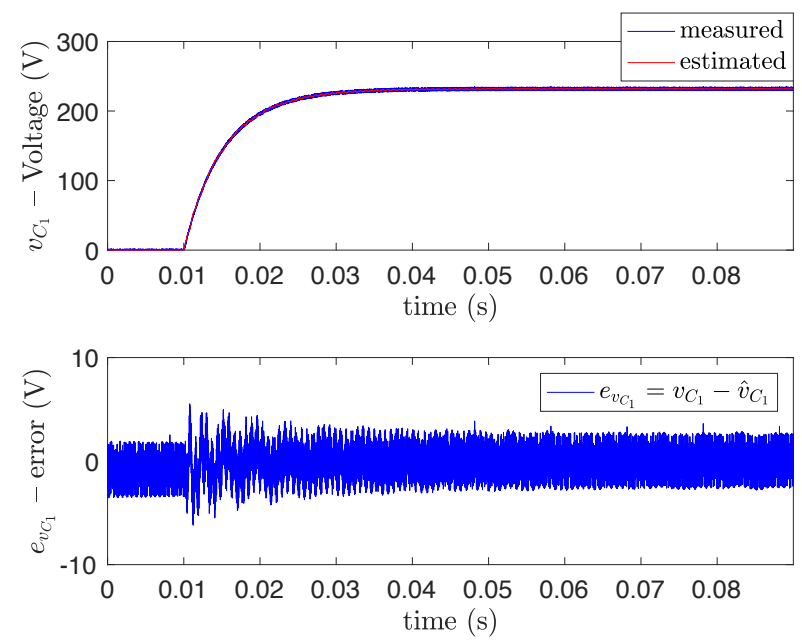

Figure 7: Estimated and measured voltage $v_{C_{1}}$, and its estimation error, during a start-up test. 

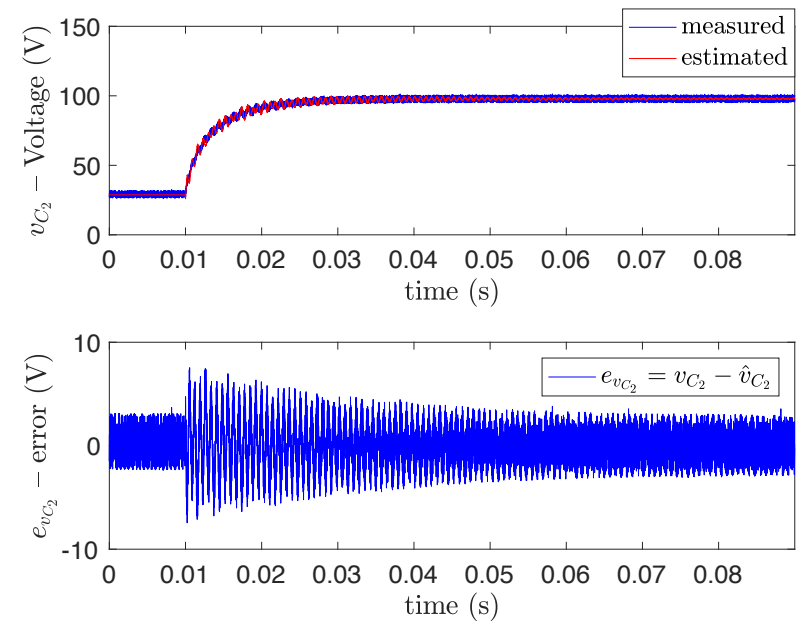

Figure 8: Estimated and measured current $v_{C_{2}}$, and its estimation error, during a start-up test.

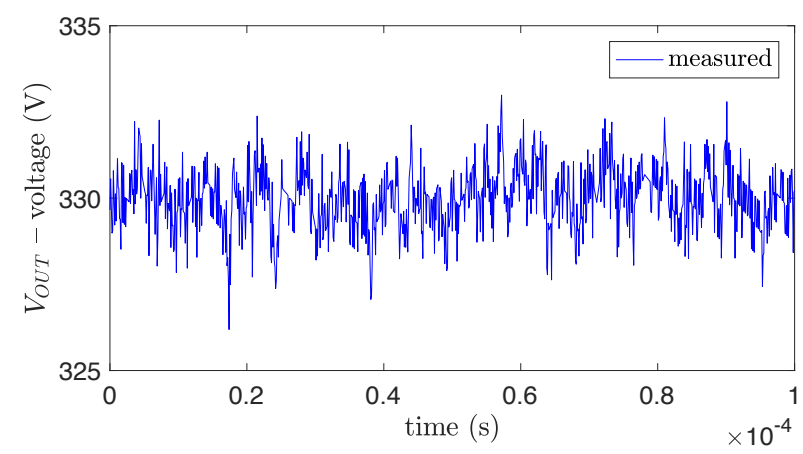

Figure 9: Output voltage at steady-state.
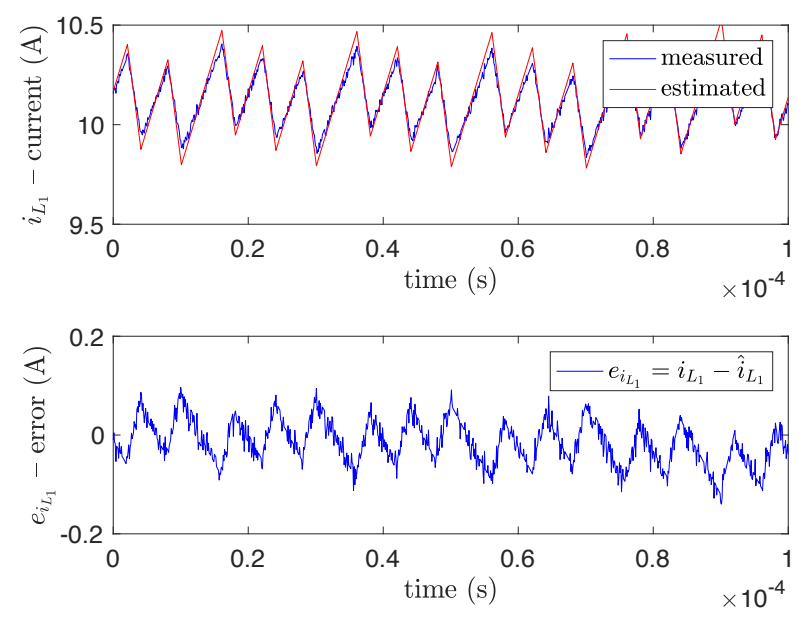

Figure 10: Estimated and measured current $i_{L_{1}}$, and its estimation error at steady-state.
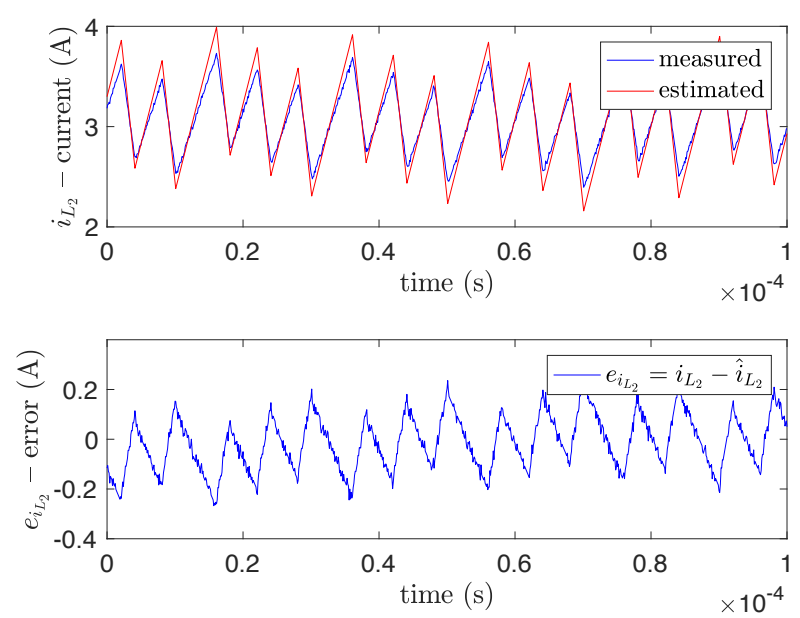

Figure 11: Estimated and measured current $i_{L_{2}}$, and its estimation error at steady-state.
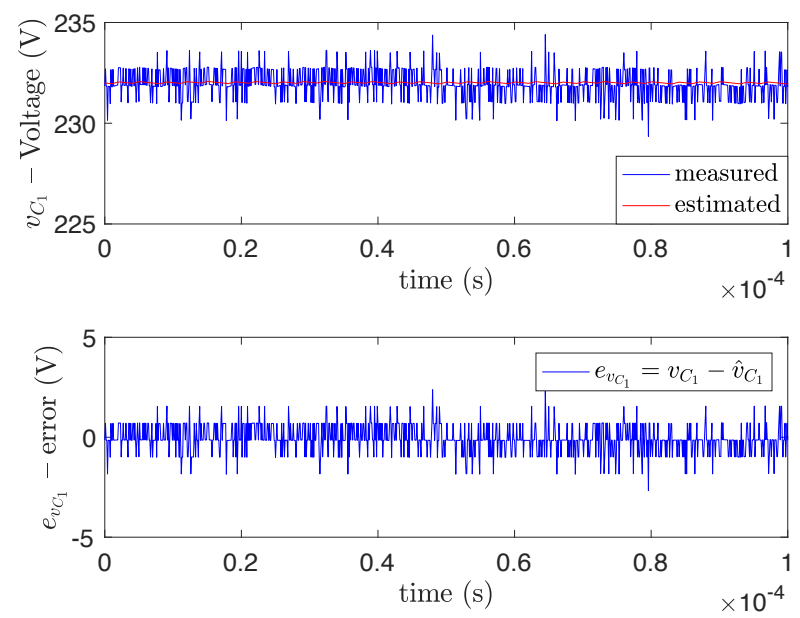

Figure 12: Estimated and measured voltage $v_{C_{1}}$, and its estimation error at steady-state.
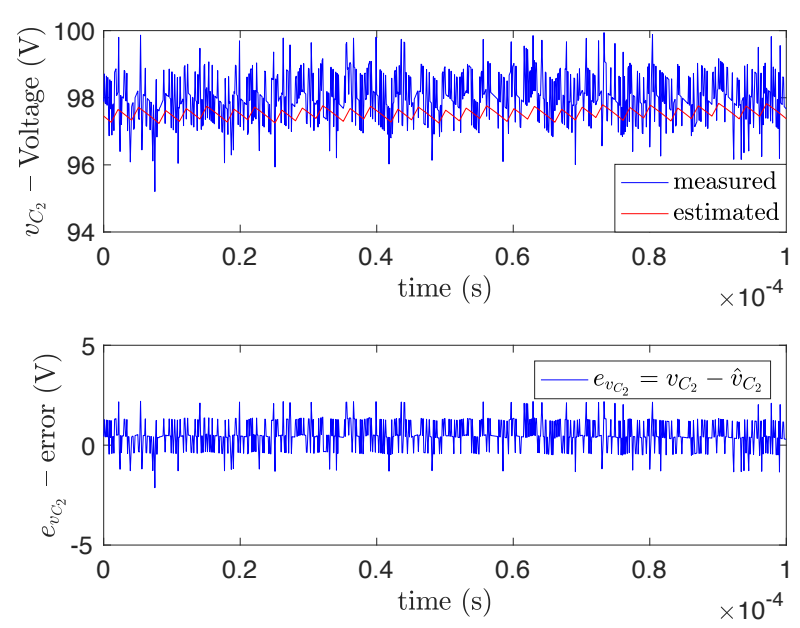

Figure 13: Estimated and measured current $v_{C_{2}}$, and its estimation error at steady-state. 


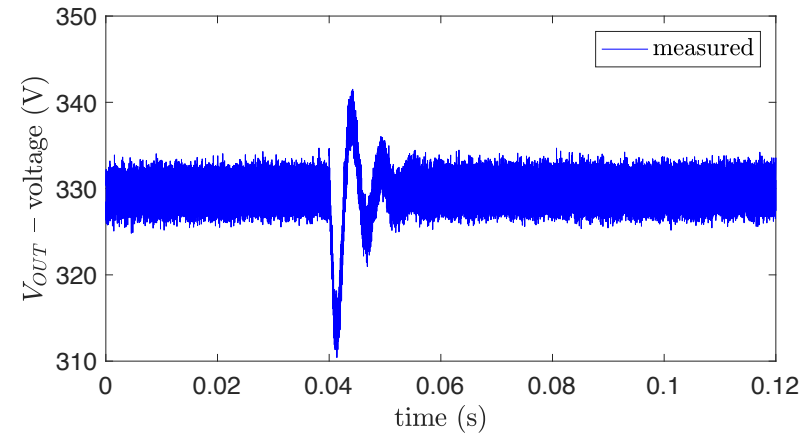

Figure 14: Output voltage during a variation of the load from $390 \Omega$ to $250 \Omega$.
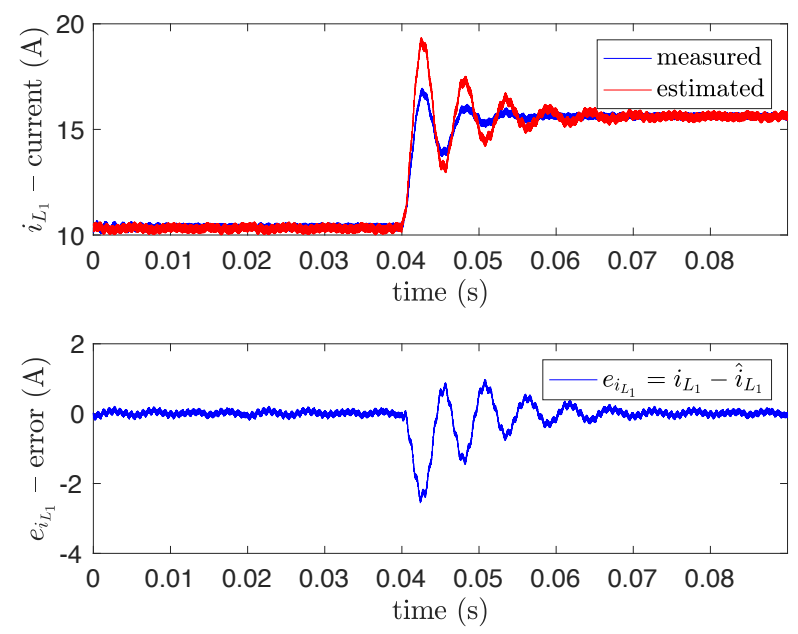

Figure 15: Estimated and measured current $i_{L_{1}}$, and its estimation error, during a variation of the load from $390 \Omega$ to $250 \Omega$.
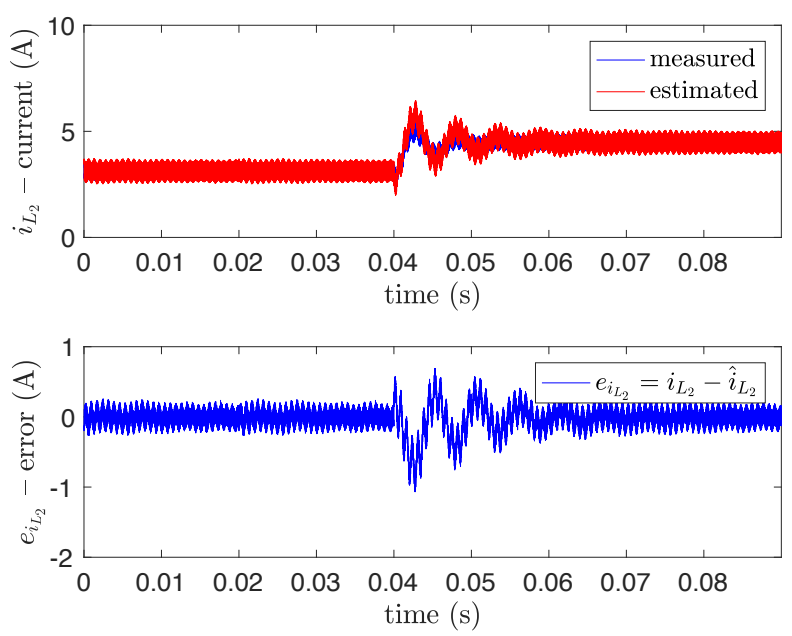

Figure 16: Estimated and measured current $i_{L_{2}}$, and its estimation error, during a variation of the load from $390 \Omega$ to $250 \Omega$.
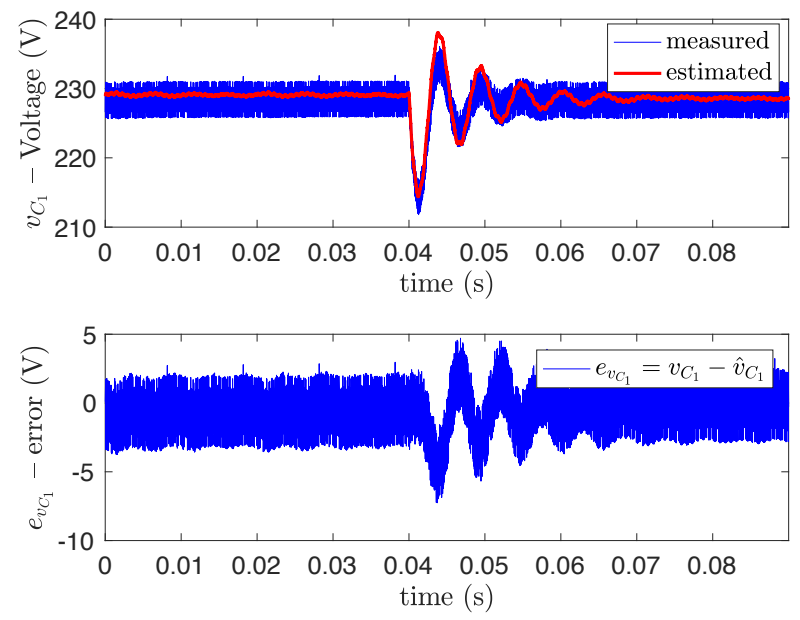

Figure 17: Estimated and measured voltage $v_{C_{1}}$, and its estimation error, during a variation of the load from $390 \Omega$ to $250 \Omega$.
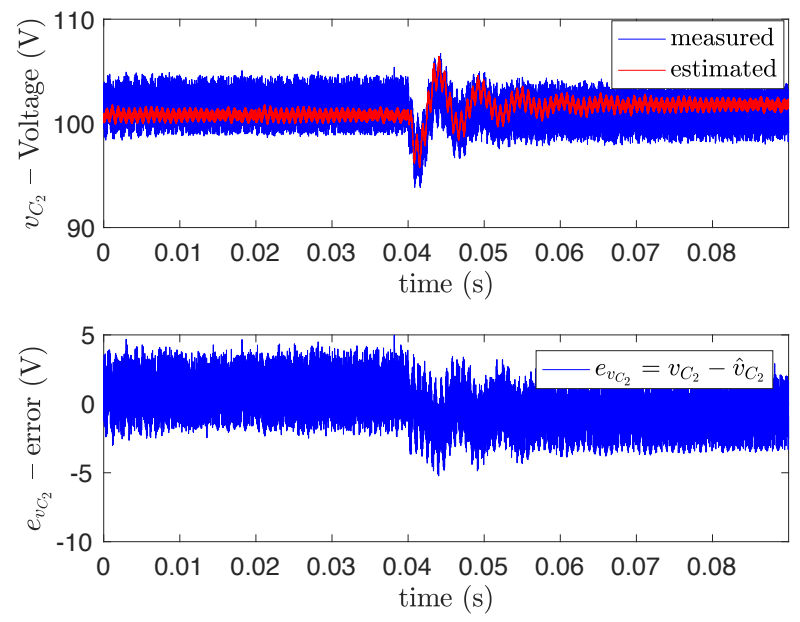

Figure 18: Estimated and measured current $v_{C_{2}}$, and its estimation error, during a variation from $390 \Omega$ to $250 \Omega$ of the load.

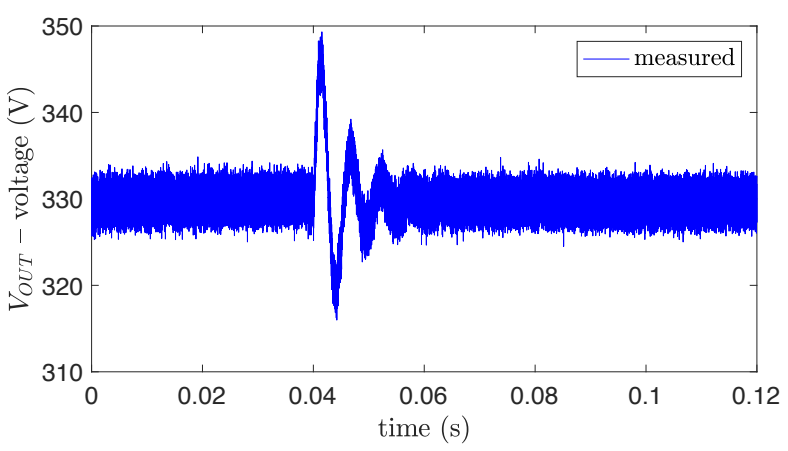

Figure 19: Output voltage during a variation from $250 \Omega$ to $390 \Omega$ of the load. 


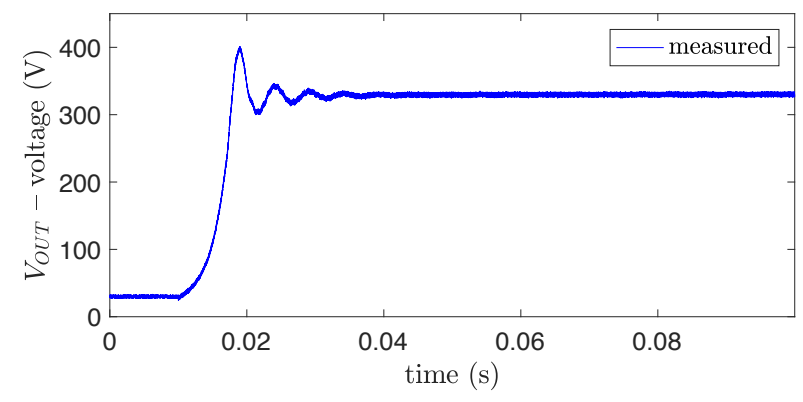

Figure 20: Output voltage during a start-up test with a PI control strategy.

Moreover, the proposed control technique is compared with a PI controller (see Fig.s 20-22), which is designed from an averaged model in continuous time. Hence, for comparison purposes, the PI controller is designed for delivering the same settling time, than the one proposed by the hybrid control strategy. However, even if the output voltage reaches the steady-state at 0.04s in both cases (see Fig.s 4 and 20), the proposed hybrid control provides a better dynamic precision because a direct control of the voltage and current trajectories in the phase-plane voltage-current is achieved (differently from PI control where only the output voltage error is considered), allowing to obtain no-inrush current at start-up (see Fig.s 5-6 and 21), as well as, a good tracking of the voltage reference (see Fig.s 7-8 and 22). Moreover, the second advantage of the proposed control algorithm is the possibility of choosing the trajectory in transient time by selecting the tuning parameter $\eta$, which allows to act directly in the switching frequency, diminishing the ripple of the inductor current. All these advantages allow to obtain a better performance using the proposed hybrid control, especially during transient times. Finally, the proposed control technique is compared with a PI controller during a step variation of the load from $390 \Omega$ to $250 \Omega$ (see Fig. 23 and14). In general, the steady state is similar. Nevertheless, the overshoot in the PI case is about $6.97 \%$, while it is about $5.76 \%$ with the proposed strategy. This light improvement in the transient time confirms the robustness of the proposed strategy with respect to parameter variations.

\section{Conclusion}

This paper proposes a hybrid control for a QBC, based on a Lyapunov matrix-based min-projection control together with the design of an observer, which estimates the state of the system, from the measurement of the input and output voltages.

Differently from classical control methods, where the controller is designed based on small-signal models, here a model based on the hybrid nature of the converter, i.e. on the continuous-time and discrete-time dynamics, is considered. Stability properties of the complete system are established using a hybrid dynamical system theory, in terms of UGAS of a bounded and closed set where both, the estimation and the tracking error converge to a neighborhood of zero, ensuring globally practically stability of these errors equal to zero. The
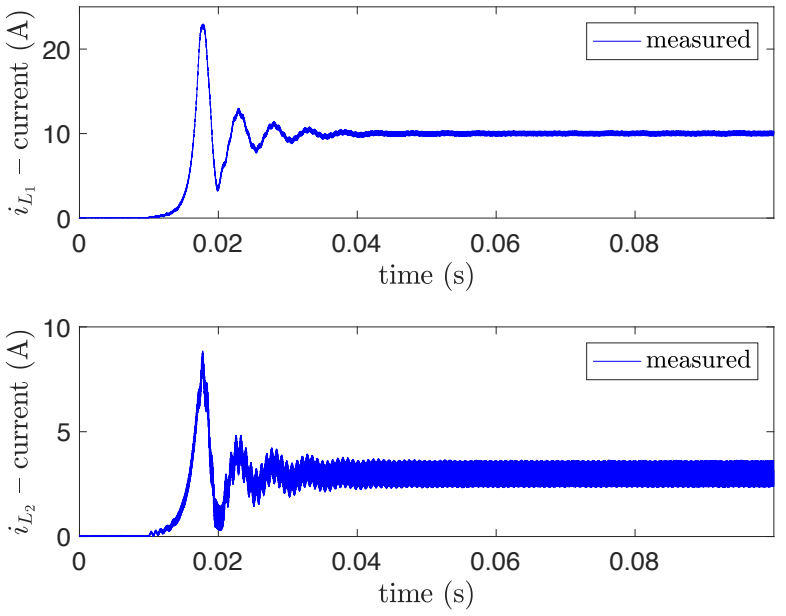

Figure 21: Measured currents $i_{L_{1}}$ and $i_{L_{2}}$, during a start-up test with a PI control strategy.
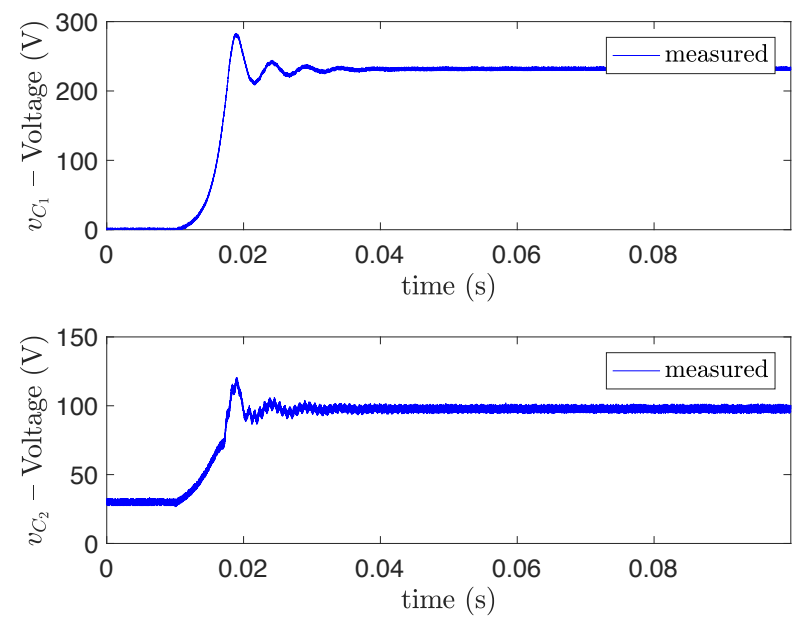

Figure 22: Measured currents $v_{C_{1}}$ and $v_{C_{2}}$, during a start-up test with a PI control strategy.

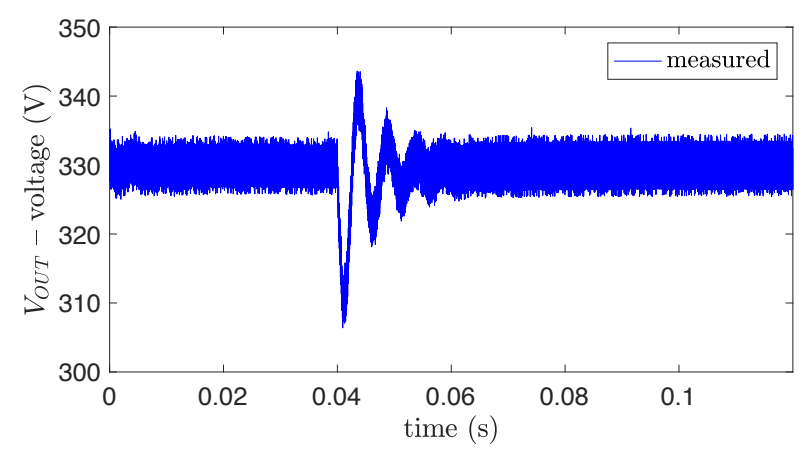

Figure 23: Output voltage during a variation of the load from $390 \Omega$ to $250 \Omega$ with a PI control strategy. 
performed experimental results show the efficiency of the proposed approach, and the capability of the observer to track the state variables with small enough errors. In particular, the experimental tests show the better performance achieved using the hybrid controller with respect to conventional control methods such as a PI controller, especially in terms of dynamic precision. Finally, this work provides theoretical and practical results to design a state observer for removing the current sensors in power converters.

\section{Acknowledgement}

This work has been partially funded under grant "HISPALIS" ANR-18-CE40-0022-01.

\section{References}

Albea Sanchez, C., Lopez Santos, O., Prada Zambrano, D., Gordillo, F., Garcia, G., 2019. On the practical stability of hybrid control algorithm with minimum dwell time for a DC-AC converter. IEEE Trans. on Control System Technology 27 (6), 2581-2588.

Al-Saffar, M. A., Ismail, E. H., 2015. A high voltage ratio and low stress DCDC converter with reduced input current ripple for fuel cell source. Renewable Energy 82, 35-43.

Albea-Sanchez, C., Garcia, G., Zaccarian, L., 2015. Hybrid dynamic modeling and control of switched affine systems: application to DC-DC converters. In: Annual Conference on Decision and Control. IEEE, pp. 2264-2269.

Alonge, F., Pucci, M., Rabbeni, R., Vitale, G., 2017. Dynamic modelling of a quadratic DC-DC single-switch boost converter. Electric Power Systems Research 152, 130-139.

Alonge, F., Rabbeni, R., Pucci, M., Vitale, G., 2015. Identification and robust control of a quadratic DC-DC boost converter by hammerstein model. IEEE Trans. on Industry Applications 51 (5), 3975-3985.

Baek, J.-W., Ryoo, M.-H., Kim, T.-J., Yoo, D.-W., Kim, J.-S., 2005. High boost converter using voltage multiplier. In: Annual Conference of IEEE Industrial Electronics Society. IEEE, pp. 567-572.

Carrasco, J. M., Franquelo, L. G., Bialasiewicz, J. T., Galván, E., PortilloGuisado, R. C., Prats, M. M., León, J. I., Moreno-Alfonso, N., 2006. Powerelectronic systems for the grid integration of renewable energy sources: A survey. IEEE Trans. on industrial electronics 53 (4), 1002-1016.

Chen, J., MaksimoviC, D., Erickson, R., 2001. Buck-boost PWM converters having two independently controlled switches. In: Power Electronics Specialists Conference. Vol. 2. IEEE, pp. 736-741.

Chen, Z., Yang, P., Zhou, G., Xu, J., Chen, Z., 2016. Variable duty cycle control for quadratic boost PFC converter. IEEE Trans. on Industrial Electronics 63 (7), 4222-4232.

Chincholkar, S. H., Chan, C.-Y., 2017. Design of fixed-frequency pulsewidthmodulation-based sliding-mode controllers for the quadratic boost converter. IEEE Trans. on Circuits and Systems II: Express Briefs 64 (1), 51-55.

Choudhury, T. R., Nayak, B., 2015. Comparison and analysis of cascaded and quadratic boost converter. In: Power, Communication and Information Technology Conference. IEEE, pp. 78-83.

García-Vite, P. M., Soriano-Rangel, C. A., Rosas-Caro, J. C., Mancilla-David, F., 2017. A DC-DC converter with quadratic gain and input current ripple cancelation at a selectable duty cycle. Renewable Energy 101, 431-436.
Goebel, R., Sanfelice, R. G., Teel, A. R., 2012. Hybrid Dynamical Systems: modeling, stability, and robustness. Princeton University Press.

Iannelli, L., Johansson, K. H., Jönsson, U. T., Vasca, F., 2008. Subtleties in the averaging of a class of hybrid systems with applications to power converters. Control Engineering Practice 16 (8), 961-975.

Leyva-Ramos, J., Ortiz-Lopez, M., Diaz-Saldierna, L., Morales-Saldana, J., 2009. Switching regulator using a quadratic boost converter for wide DC conversion ratios. IET Power Electronics 2 (5), 605-613.

Liberzon, D., 2012. Switching in systems and control. Springer Science \& Business Media.

Liu, J., Laghrouche, S., Harmouche, M., Wack, M., 2014. Adaptive-gain second-order sliding mode observer design for switching power converters. Control Engineering Practice 30, 124-131.

López-Santos, O., Martínez-Salamero, L., García, G., Valderrama-Blavi, H., Mercuri, D. O., 2013. Efficiency analysis of a sliding-mode controlled quadratic boost converter. IET Power Electronics 6 (2), 364-373.

Lopez-Santos, O., Martinez-Salamero, L., Garcia, G., Valderrama-Blavi, H., Sierra-Polanco, T., 2015. Robust sliding-mode control design for a voltage regulated quadratic boost converter. IEEE Trans. on Power Electronics 30 (4), 2313-2327.

Lopez-Santos, O., Martinez-Salamero, L., Garcia, G., Valderrama-Blavi, H., Zambrano-Prada, D., 2017. Steady-state analysis of inductor conduction modes in the quadratic boost converter. IEEE Trans. on Power Electronics 32 (3), 2253-2264.

Luo, F., Ye, H., 2004. Positive output cascade boost converters. IEE Electric Power Applications 151 (5), 590-606.

Mariethoz, S., Almér, S., Bâja, M., Beccuti, A. G., Patino, D., Wernrud, A., Buisson, J., Cormerais, H., Geyer, T., Fujioka, H., et al., 2010. Comparison of hybrid control techniques for buck and boost DC-DC converters. IEEE Trans. on control systems technology 18 (5), 1126-1145.

Morales-Saldana, J. A., Galarza-Quirino, R., Leyva-Ramos, J., CarbajalGutierrez, E., Ortiz-Lopez, M., 2007. Multiloop controller design for a quadratic boost converter. IET Electric Power Applications 1 (3), 362-367.

Morales-Saldaña, J. A., Loera-Palomo, R., Palacios-Hernández, E., GonzálezMartínez, J. L., 2014. Modelling and control of a DC-DC quadratic boost converter with $r^{2} p^{2}$. IET Power Electronics 7 (1), 11-22.

Olalla, C., Queinnec, I., Leyva, R., El Aroudi, A., 2011. Robust optimal control of bilinear DC-DC converters. Control Engineering Practice 19 (7), 688699.

Patidar, K., Umarikar, A. C., 2015. High step-up converters based on quadratic boost converter for micro-inverter. Electric Power Systems Research 119, 168-177.

Prieur, C., Teel, A. R., Zaccarian, L., 2014. Relaxed persistent flow/jump conditions for uniform global asymptotic stability. IEEE Trans. on Automatic Control 59 (10), 2766-2771.

Sanchez, C. A., Garcia, G., Hadjeras, S., Heemels, W., Zaccarian, L., 2019. Practical stabilization of switched affine systems with dwell-time guarantees. IEEE Transactions on Automatic Control 64 (11), 4811-4817.

Sferlazza, A., Albea-Sanchez, C., Martínez-Salamero, L., Garcia, G., Alonso, C., 2019. Min-type control strategy of a DC-DC synchronous boost converter. IEEE Transactions on Industrial Electronics 67 (4), 3167-3179.

Wang, F., 2018. A novel quadratic boost converter with low current and voltage stress on power switch for fuel-cell system applications. Renewable energy $115,836-845$.

Zhao, Q., Lee, F. C., 2003. High-efficiency, high step-up DC-DC converters. IEEE Trans. on Power Electronics 18 (1), 65-73. 